\title{
A Case Report of Necrotizing Eosinophilic Folliculitis Occurring After Rhus Ingestion
}

\author{
En Hyung Kim', Dae Hwi Eun $\mathbb{D}^{2}$, ji Yeoun Lee ${ }^{2}$ \\ 'Department of Dermatology, Bundang Jesaeng General Hospital, Seongnam, Gyeonggi, Korea; ${ }^{2}$ Department of Dermatology, College of Medicine, \\ Chungbuk National University, Cheongju, Korea \\ Correspondence: Ji Yeoun Lee, Department of Dermatology, College of Medicine, Chungbuk National University, Chungdae-ro I, Seowon-Gu, \\ Cheongju, Chungbuk, 28644, Korea, Tel +82-43-269-6385, Fax +82-43-266-1698, Email jyl@chungbuk.ac.kr
}

\begin{abstract}
Necrotizing eosinophilic folliculitis is known as a rare variant of eosinophilic folliculitis (EF). It differs from classic EF because of its necrotizing ulcerative clinical course. We report a case of necrotizing eosinophilic folliculitis accompanied by pathergic phenomenon that may be the result of a delayed type hypersensitivity reaction occurring after Rhus consumption. Painful pyodermic plaques developed in a 48-year-old man, over a 5-day period, which was associated with facial edema. The lesions appeared 2 days after ingestion of Rhus. His face showed multiple painful pruritic papules that coalesced into crusted plaque accompanied by hemorrhagic eschars. At the periphery of the plaques, multiple erythematous papules and pustules were noticed. Prominent tissue eosinophilia was noticed from the skin biopsy samples. Also, there were areas of follicular mucinosis and eosinophilic folliculitis as well as hemorrhagic scale crust over the surface. Based on the clinical and histologic findings, the patient was diagnosed as necrotizing eosinophilic folliculitis occurring after Rhus ingestion. The lesions rapidly cleared after starting treatment with systemic steroid and dapsone. Clinicians should be aware that necrotizing eosinophilic folliculitis may occur after Rhus contact or consumption, in order to diagnose and treat patients promptly and reduce patient discomfort and skin scarring.
\end{abstract}

Keywords: necrotizing eosinophilic folliculitis, Ofuji's disease, Rhus dermatitis

\section{Introduction}

Rhus (Toxicodendron succedaneum) is used in Korea as a medicinal plant to boost immunity. The leaves, roots and bark of the plant are boiled to make extracts, and then ingested per se or added when cooking chicken at home or specialized restaurants. Also, in oriental medicinal preparations, dried Rhus tree is used as an ingredient. In western countries, it can be found in private gardens and distribution of its seeds by birds can lead to it becoming established in public or urban areas. It is one of the most common plant families to cause allergic contact dermatitis worldwide. ${ }^{1,2}$ Rhus contact dermatitis (RCD) is usually manifested as eczema, pompholyx, symmetrical maculopapular rash, urticaria, erythema multiforme and rarely vasculitis or fixed drug eruption. ${ }^{3}$

Eosinophilic folliculitis (EF) was first described as an idiopathic eruption of sterile pustules, papules, and plaques, preferentially involving the face, trunk, and extremities of men in the third and fourth decades of life. ${ }^{4}$ It is broadly divided into five major groups: (i) classical EF, or Ofuji disease, (ii) HIV-associated EF, (iii) infantile EF, (iv) cancer-associated EF, and (v) medication-associated EF. ${ }^{5}$ Necrotizing eosinophilic folliculitis is known as a rare variant of eosinophilic EF. It is associated with atopy and characterized histopathologically by dermal neutrophilia, eosinophilic vasculitis, ulceration and marked follicular destruction. ${ }^{6}$ Here, we report a 48-year-old man who developed necrotizing eosinophilic folliculitis after consuming Rhus. His pathergy reaction was positive, which may indicate a delayed type hypersensitivity reaction to Rhus ingestion.

\section{Case}

A 48-year-old man presented with painful pyodermic plaques with edema localized to his face (Figure 1A). The lesions developed 2 days after Rhus consumption. The patient had previous history of Rhus chicken consumption. He worked as 

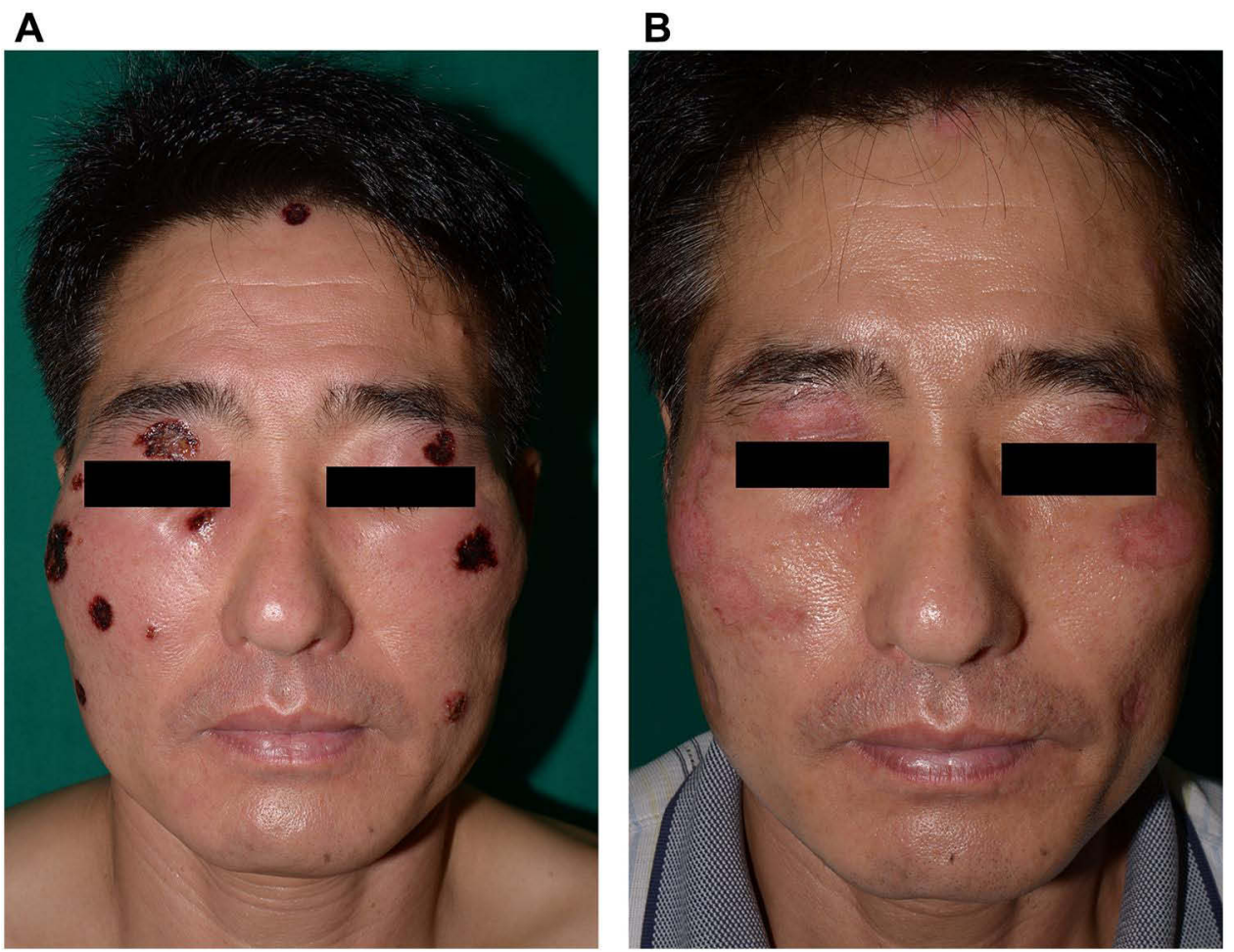

Figure I (A) Multiple pyodermic plaques with hemorrhagic eschar on the face. (B) The lesions cleared up leaving mild scarring 10 days later.

a construction site supervisor; however, he had no exposure to halogen. He had been healthy without any systemic diseases. On physical examination, there were numerous tender, indurated plaques on the patient's face and neck area. Several erythematous papular and pustular lesions were seen at the periphery of the plaques. The eruption was not associated with fever or other general symptoms. Full blood count, liver and renal function tests, fasting sugar, C-reactive protein and urinalysis were all within normal range. Cytoplasmic-antineutrophil cytoplasmic antibodies (c-ANCA), perinuclear-antineutrophil cytoplasmic antibodies (p-ANCA), antinuclear antibody (ANA) tests were also unremarkable. Evaluation for infection including serology for venereal disease research laboratory (VDRL) and human immunodeficiency virus (HIV) were negative, and cultures of peripheral blood and skin pustules were negative. Tzanck smear and fungal testing from skin lesions did not show any abnormalities. Routine stool tests on common pathogen and parasite screen were negative.

Three skin biopsies were taken from the face. Light microscopy findings revealed prominent superficial and deep lymphocytic inflammatory reaction with numerous eosinophilic and neutrophilic infiltrate in the follicular infundibulum and follicular spongiosis (Figure 2A). The epidermis was covered with serum crust with underlying neutrophilic and eosinophilic dermolysis. Eosinophilic abscesses were noticed in follicular infundibular orifices. Exocytosis of lymphocytes and eosinophils were noticed in the hair follicles (Figure 2B). There was a moderately dense lymphocytic inflammation, which extended around the superficial and mid-dermal vessels. The samples showed indications of vasculitic change, such as mild luminal intramural fibrin deposition and migration of eosinophils and neutrophils through the vessel wall. Slight perivascular dermal necrosis was noticed (Figure 2C). Periodic acid-Schiff stains for fungi were negative, and there was no evidence of viral cytopathic changes such as herpes infection or extravascular necrotizing granulomas.

Based on the clinical and histological findings, the patient was diagnosed with necrotizing eosinophilic folliculitis and was treated with intravenous injections of $5 \mathrm{mg}$ dexamethasone twice a day along with oral dapsone at a dose of $50 \mathrm{mg}$. Hydrogel (Intrasite gel $25 \mathrm{mg}$, Smith \& Nephew (C) was used as dressing material. The lesions improved rapidly within 10 days of commencing treatment and drug dose was tapered over a 2-week period. The lesions cleared up leaving mild scarring and there was no recurrence over a 1-year follow-up period (Figure 1B). 
A

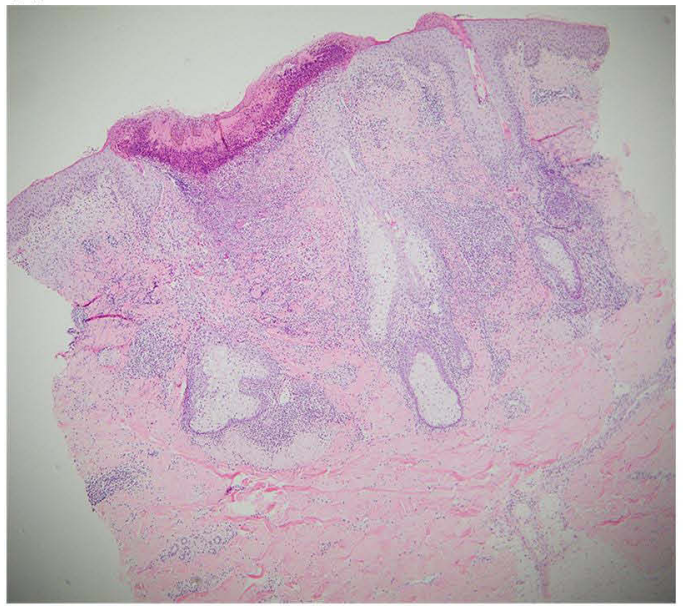

B

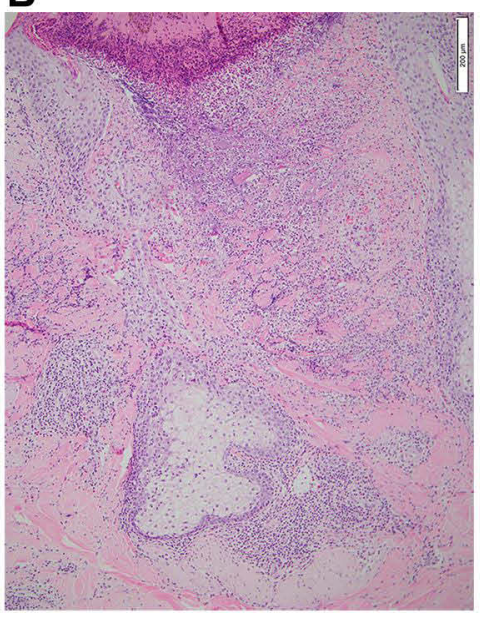

C

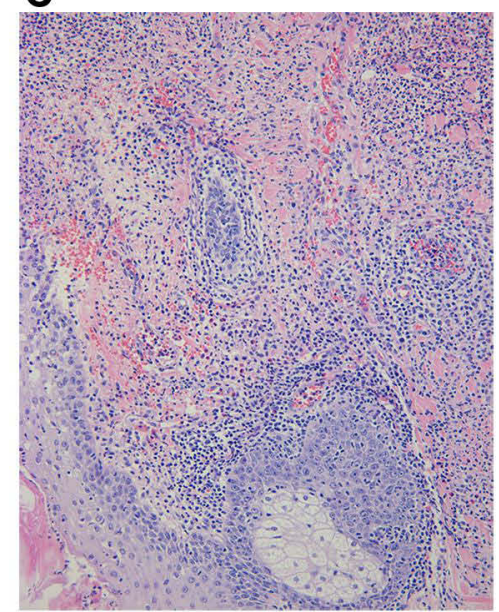

Figure 2 (A) Skin biopsy showing perivascular and perifollicular inflammatory cell infiltration with numerous eosinophilic and neutrophilic infiltrate in the follicular infundibulum and follicular spongiosis. (Hematoxylin and eosin; $x 40$ ), (B) The follicles showed exocytosis of both lymphocytes and eosinophils. Epidermal necrosis with underlying neutrophilic and eosinophilic dermolysis was also noticed. (Hematoxylin and eosin; xI00), (C) Vasculitic changes with migration of eosinophils and neutrophils through the vessel wall with mild luminal intramural fibrin deposition. (Hematoxylin and eosin; $x$ 200).

\section{Discussion}

Sometimes pathergic phenomenon occurs in EF patients with an atopic background and the term "necrotizing eosinophilic folliculitis" was proposed to describe this condition. ${ }^{6}$ The clinical manifestations of EF may be the result of an abnormal T helper (Th) 2-type immune response to a follicular antigen. Necrotizing eosinophilic folliculitis has been reported in patients with a range of immune dysregulatory states, such as atopy. It is characterized by significant tissue destruction, which differentiates it from EF. ${ }^{6,7}$ T-cell dysregulation is an important factor in the pathogenesis of necrotizing eosinophilic folliculitis. ${ }^{6}$ Immune triggers such as bacteria can lead to an exaggerated type IV immune reaction in a Th2 cytokine-rich environment as in atopy. This may result in the pathognomonic destructive folliculocentric process in NEF. ${ }^{6}$ In atopy, cytokines like interleukin (IL)-4 and IL-5 are elevated. These cytokines may stimulate eosinophil proliferation and induce the infiltrates seen in necrotizing eosinophilic folliculitis. ${ }^{8}$ In this case, the patient had consumed Rhus, which is well known for its type-IV delayed hypersensitivity reaction. After contact, macrophages present the antigen to Th-cells, then the sensitized Th-cells start to release cytokines and chemokines. The excessive inflammatory response may cause damage to the keratinocytes resulting in epithelial tissue disruptions. ${ }^{9}$ Thus, the necrotizing ulcerative features noticed in this patient may have been initiated by a delayed-type hypersensitivity reaction to Rhus and aggravated by miscellaneous causes as shown by his positive pathergic reaction.

As our patient shared features with a number of conditions, it was difficult to diagnose and find proper treatment. Our differential diagnosis included Sweet's syndrome, pyoderma faciale, eczema herpeticum, superficial granulomatous pyoderma and halogenoderma. Sweet's syndrome presents as reddish blue or violet plaques or nodules that uncommonly are confined to the face. Lesions may be studded with pustules. Skin biopsy reveals dense, diffuse neutrophilic infiltrate in the reticular dermis with leukocytoclasis, which was not a prominent feature in our case. ${ }^{10}$ Pyoderma faciale presents as tender follicular papules and pustules, nodules with explosive onset. Biopsy reveals perifollicular and perisebaceous gland abscesses with neutrophils rather than eosinophils. ${ }^{12}$ Eczema herpeticum may begin as sudden outbreaks of painful, edematous, often crusted or hemorrhagic vesicles, pustules or erosions. Umblicated vesiculopustules progress to punched-out erosions. Skin biopsy shows changes characteristic of herpes virus infection, such as multinucleated epithelial cells and ballooning degeneration of keratinocytes. ${ }^{10}$ Superficial granulomatous pyoderma is a rare variant of pyoderma gangrenosum. Lesions usually appear as a single, well-defined non-tender, superficial ulcer with exophytic or vegetating clean granulations. ${ }^{11}$ Histopathologically, there is reactive epidermal hyperplasia accompanied by neutrophil-rich inflammation and focal granuloma formation. The ulcers show a characteristic layered granuloma. However in our patient, the distribution and multiplicity of the lesions, painful 
feature and histologic findings of eosinophil-rich vasculitic change, tissue eosinophilia favor necrotizing eosinophilic folliculitis rather than superficial granulomatous pyoderma or pyoderma gangrenosum. Halogenoderma is characterized by vesicular, pustular, hemorrhagic, ulcerative lesions but is usually not confined to the face. In histopathology, pseudoepitheliomatous hyperplasia is observed and often, intraepidermal abscesses form with neutrophils and eosinophils. ${ }^{10}$ However, exposure history to halogen-containing drugs or substances is necessary.

The histological findings were the ultimate key to diagnosis, but even after diagnosis it was still challenging to choose a treatment that would minimize skin scarring and terminate the inflammatory process. Treatment with dapsone, prednisone, cyclophosphamide, indomethacin and cephalexin has been reported to be successful for necrotizing eosinophilic folliculitis. ${ }^{6}$ Our patient showed rapid response to systemic steroid and dapsone therapy.

\section{Conclusion}

There have been worldwide reports of RCD. Clinicians should be aware that besides the well-known features of RCD, such as maculopapular rash or eczema, necrotizing eosinophilic folliculitis may occur after Rhus contact or consumption. Careful clinicopathological correlation is needed for prompt diagnosis and proper treatment to reduce patient discomfort and skin scarring.

\section{Ethics Statement}

The publications of images were included in the patient's consent for publication of the case. Institutional approval has been obtained to publish the case details.

\section{Consent Statement}

The authors certify that they have obtained all appropriate patient consent forms. The patient signed a consent form for the publication of the case details and images.

\section{Acknowledgments}

The abstract of this paper was presented at the 2018 JAAD Conference as a poster presentation with interim findings. The poster's abstract was published in "Poster Abstracts" in September 2018 Volume 79 Issue 3 Supplement 1 AB1-AB420 Poster Abstracts: DOI: https://doi.org/10.1016/j.jaad.2018.05.815.

\section{Disclosure}

The authors report no conflicts of interest in this work.

\section{References}

1. Gach JE, Tucker W, Hill VA. Three cases of severe Rhus dermatitis in an English primary school. J Eur Acad Dermatol Venereol. 2006;20 (2):212-213. doi:10.1111/j.1468-3083.2006.01414.x

2. Robin JS, Michael GB, Leo JS. Dermatitis due to Toxicodendron plants: a common occurrence during autumn. N Z Med J. 2017;130(1451):82-83.

3. Oh SH, Haw CR, Lee MH. Clinical and immunologic features of systemic contact dermatitis from ingestion of Rhus (Toxicodendron). Contact Dermatitis. 2003;48(5):251-254. doi:10.1034/j.1600-0536.2003.00103.x

4. Ofuji S, Ogino A, Horio T, et al. Eosinophilic pustular folliculitis. Acta Derm Venereol. 1970;50(3):195-203.

5. Nomura T, Katoh M, Yamamoto Y, et al. Eosinophilic pustular folliculitis: a proposal of diagnostic and therapeutic algorithms. J Dermatol. 2016;43 (11):1301-1306. doi:10.1111/1346-8138.13359

6. Magro CM, Crowston AN. Necrotizing eosinophilic folliculitis: a novel manifestation of the atopic diathesis? Int J Dermatol. 2000;39(9):672-772. doi:10.1046/j.1365-4362.2000.00973.x

7. Magro CM, Crowston AN. Eosinophilic pustular follicular reaction: a paradigm of immune dysregulation. Int J Dermatol. 1994;33(3):172-178. doi:10.1111/j.1365-4362.1994.tb04943.x

8. Soeprono FF, Schinella RA. Eosinophilic folliculitis in patients with the acquired immunodeficiency syndrome. J Am Acad Dermatol. 1986;14 (6):1020-1022. doi:10.1016/S0190-9622(86)70126-6

9. Dispenza MC. Classification of hypersensitivity reactions. Allergy Asthma Proc. 2019;40(6):470-473. doi:10.2500/aap.2019.40.4274

10. Steven K. Necrotizing eosinophilic folliculitis with mucinosis. Australas J Dermatol. 2003;44(4):298-301. doi:10.1046/j.1440-0960.2003.00014.x

11. Wilson-Jones E, Winkelmann R. Superficial granulomatous pyoderma: a localized vegetative form of pyoderma gangrenosum. $J$ Am Acad Dermatol. 1988;18:511-521. doi:10.1016/S0190-9622(88)70074-2

12. Plewig G, Jansen T, Kligman AM. Pyoderma faciale. A review and report of 20 additional cases: is it rosacea? Arch Dermatol. 1992;128 (12):1611-1617. doi:10.1001/archderm.1992.04530010049007 


\section{Publish your work in this journal}

Clinical, Cosmetic and Investigational Dermatology is an international, peer-reviewed, open access, online journal that focuses on the latest clinical and experimental research in all aspects of skin disease and cosmetic interventions. This journal is indexed on CAS. The manuscript management system is completely online and includes a very quick and fair peer-review system, which is all easy to use. Visit http://www. dovepress.com/testimonials.php to read real quotes from published authors.

Submit your manuscript here: https://www.dovepress.com/clinical-cosmetic-and-investigational-dermatology-journal 